\title{
RELACIÓN ENTRE ESTRATEGIAS DE AFRONTAMIENTO, SÍNDROMES CLÍNICOS Y TRASTORNOS DE PERSONALIDAD EN PACIENTES ESQUIZOFRÉNICOS CRÓNICOS
}

\author{
M. DOLORES FERNÁNDEZ y MIGUEL A. DÍAZ \\ Unidad de Rehabilitación de Área (URA), Puerto de Santa María, Cádiz
}

(Aceptado en mayo de 2001)

\begin{abstract}
El objetivo del trabajo fue estudiar si los estilos de afrontamiento de pacientes esquizofrénicos se relacionaban con los trastornos de personalidad y los síndromes clínicos del Inventario Clínico Multiaxial de Millon (MCMI-II), así como determinar cuál era el perfil obtenido por pacientes esquizofrénicos mediante el MCMI-II. Los resultados indican relaciones significativas entre las estrategias de afrontamiento negativas (emoción y evitación) y aquellas escalas de personalidad en las cuales los sujetos obtienen puntuaciones significativas. Así mismo, las estrategias de afrontamiento centradas en la tarea se relacionan con un menor grado de patología.
\end{abstract}

Palabras clave: Personalidad, estrategias de afrontamiento, esquizofrenia, Inventario Clínico Multiaxial de Millon.

\section{Relationship among ways of coping, clinical syndromes, an personality disorders in chronic schizophrenics patients}

\begin{abstract}
The aim of this paper has been to study whether coping styles in schizophrenics are related to personality syndromes and disorders as measured Millon Multiaxial Clinical Inventory (MCMI-II) and the characteristic profile of chronic schizophrenics on this test. Results show an important relationship between negative coping strategies (emotion and avoidance) and those subscales with high scores and also between task-centered coping strategies and low psychopathology.
\end{abstract}

Key words: Personality, coping strategies, schizophrenics, Millon Inventory.

\section{INTRODUCCIÓN}

Según la definición del Manual Diagnóstico y Estadístico de los Trastornos Mentales (DSM-IV) (Asociación Americana de Psiquiatría, 1994), los trastornos de la personalidad implican inflexibles y desadaptativos rasgos de personalidad que causan un malestar subjetivo o un deterioro funcional significativo. Este deterioro supone dificultades en la regulación de los afectos, relaciones con los otros, consecución de metas y manejo del estrés (Beck y Freeman, 1990; Millon, 1981). El modelo transaccional del estrés (Lazarus y Folkman, 1984) ofrece un marco de trabajo para el estudio de las relaciones que se pueden establecer entre las dificultades derivadas de los trastornos de personalidad y las distintas estrategias de afrontamiento que utilizan los sujetos. Según Lazarus y Folkman (1980) el afrontamiento implica todos los esfuerzos cognitivos y conductuales para manejar las demandas externas o internas que son evaluadas como algo que grava o excede los recursos de la persona, es decir, para el manejo de una situación estresante y el estado emocional que genera.

Para Endler y Parker (1990) existen principalmente tres tipos de afrontamiento: (1) Afrontamiento orientado hacia la 
tarea: este tipo de afrontamiento se centra en resolver el problema de manera lógica, centrándose en las soluciones y en la elaboración de planes de acción. (2) Afrontamiento orientado hacia la emoción: este tipo de afrontamiento se centra en respuestas emocionales, autopreocupaciones y reacciones fantásticas. (3) Afrontamiento orientado hacia la evitación: este tipo de afrontamiento se centra en buscar otras situaciones que nos hagan evadirnos del problema, evitarlo. Por ejemplo, visitar a un amigo (evitación- diversión) o irse a ver escaparates (evitación-distracción).

Hay que decir que las diferentes estrategias de afrontamiento que el sujeto utiliza en un momento determinado influyen en que la persona viva la situación como estresante o, por el contrario, que pueda suprimir el estado emocional de estrés y ayudar de esta forma a la adaptación del sujeto a su medio. Siguiendo a Lazarus y Folkman (1980), la consideración del afrontamiento como proceso implica asumir que dicho término se emplea independientemente de que los resultados sean eficaces o ineficaces. No existen procesos de afrontamiento universalmente buenos o malos; ello depende de múltiples factores como la persona, el tipo específico de encuentro (relación entre la persona y el estresor), etc. No obstante, el análisis lógico de la situación estresante y la elaboración de planes de acción se relaciona frecuentemente con resultados positivos.

La asociación entre afrontamiento y trastornos de la personalidad ha sido poco estudiada. No obstante, algunos trabajos sugieren que cuando estos trastornos están presentes existe una reducida utilización de estrategias de afrontamiento positivas y una mayor predominancia de estrategias negativas (Vollrath, Alnaes y Torgersen, 1994; Kruedelbach, McCormik, Schulz y Grueneich, 1993). Las investigaciones se han centrado en mayor medida en el estudio de la rela- ción entre afrontamiento y síndromes clínicos, especialmente depresión y ansiedad, obteniéndose resultados similares a los encontrados en los trastornos de personalidad (Vollrath, Torgersen, Alnaes, 1998).

Sin embargo, son muchas las investigaciones que han estudiado la relación entre afrontamiento $y$ esquizofrenia (Bechodolf, Halve, Schultze-Lutter y Klosterkotter, 1998; Mcnally y Goldber, 1997; Middelboe y Mortensen, 1997; Van der Bosch y Rombouts, 1997; Wiedl y Schottner, 1991). Dada la frecuente comorbilidad entre la esquizofrenia y otros trastornos ( depresión, ansiedad, trastornos relacionados con sustancias, trastornos de la personalidad ) (APA, 1994) sería importante determinar qué estrategias de afrontamiento utilizadas por los pacientes esquizofrénicos se relacionan en mayor medida con los trastornos de personalidad y otros síndromes clínicos. Por ello, el objetivo del presente trabajo es estudiar si los estilos de afrontamiento de pacientes esquizofrénicos se relacionan con los trastornos de la personalidad y los síndromes clínicos, tal y como son evaluados con el Inventario Clínico Multiaxial de Millon (MCMI-II), así como determinar cuál es el perfil obtenido por esquizofrénicos crónicos en este inventario.

\section{MÉTODO}

\section{Participantes}

Se ha utilizado como muestra original un grupo de 36 pacientes que reciben actualmente tratamiento en la Unidad de Rehabilitación de Área (URA) del Puerto de Santa María (Cádiz).

En la corrección del MCMI-II se obtuvieron seis cuestionarios invalidados por lo que la muestra resultante fue de 30 sujetos. Sus edades oscilaban en un ran- 
go de 12 (mínimo de 20 y máximo de 42), con una media de edad $\pm \mathrm{DT}=31,47 \pm$ 6,94 años. El $20 \%$ eran mujeres y el $80 \%$ hombres.

Todos los pacientes poseían el diagnóstico de esquizofrenia. El $60 \%$ de tipo paranoide, el $26,7 \%$ de tipo indiferenciado y el $13,3 \%$ de tipo desorganizado. Todos realizaban tratamiento farmacológico con antipsicóticos siendo su situación clínica estable. En relación a los años de evolución, oscilaban en un rango de 16 (mínimo de 2 y máximo de 18), con una media de evolución $\pm \mathrm{DT}=7,7 \pm$ 4,57 años.

Para valorar el grado de deterioro se utilizó la Escala de Discapacidad establecida por la Organización Mundial de la Salud en 1992 (CIE 10) (World Health Organization, 1992). De acuerdo con una escala tipo Likert de 6 puntos que oscila desde 0 (ninguna discapacidad) hasta 5 (discapacidad máxima), se evalúan cuatro áreas específicas de funcionamiento: cuidado personal, ocupacional, familiar y contexto social amplio. En relación al cuidado personal, se obtuvo una media de $\pm \mathrm{DT}=$ $2 \pm 1,12$, en el funcionamiento ocupacional de $\pm \mathrm{DT}=2,76 \pm 0,76$, en el familiar de $\pm \mathrm{DT}=2.16 \pm 0,76$ y por último en el contexto social amplio de $\pm \mathrm{DT}=3 \pm 0,68$.

\section{Instrumentos}

Para registrar el tipo de estrategias de afrontamiento se utilizó el Inventario de Coping para Situaciones Estresantes (CISS) (Endler y Parker, 1990) (adaptación española de Sánchez Elvira, 1996). Está compuesto de 48 items, respecto a los cuales la persona ha de decidir, de acuerdo con una escala tipo Likert de 5 puntos que oscila desde 1 (nunca) hasta 5 (siempre), la frecuencia con que utiliza cada una de las estrategias. Permite evaluar tres tipos de estrategias de afrontamiento: orientado a la tarea, orientado a la emoción y orien- tado a la evitación; este último tipo de afrontamiento se divide en evitación-distracción y evitación-diversión.

Se administró el Inventario Clínico Multiaxial de Millon (MCMI-II) (Millon, 1987) (adaptación española de Ávila Espada, 1998). Consta de 175 items con formato de respuesta verdadero- falso, que ocupa un tiempo de administración relativamente bajo (de 15 a 25 minutos). Está compuesto de 26 escalas: 4 de fiabilidad y validez (Validez, Sinceridad, Deseabilidad y Alteración); 10 escalas básicas de personalidad (Esquizoide, Fóbica, Dependiente, Histriónica, Narcisista, Antisocial, Agresivo-Sádica, Compulsiva, Pasivo-Agresiva y Autodestructiva-Masoquista); 3 escalas de personalidad patológica (Esquizotípica, Límite y Paranoide); 6 síndromes clínicos de gravedad moderada (Ansiedad, Histeriforme, Hipomanía, Distimia, Abuso de Alcohol y Abuso de las Drogas); y 3 síndromes clínicos de gravedad severa (Pensamiento psicótico, Depresión mayor y Trastorno delirante).

\section{Tratamiento estadístico}

Se calcularon correlaciones entre cada estrategia de afrontamiento y las distintas escalas del MCMI-II. Así mismo, se realizaron análisis de regresión múltiple con el método de pasos sucesivos. Las variables dependientes introducidas fueron las escalas de personalidad y síndromes clínicos del MCMI-II y las variables independientes los tipos de afrontamiento evaluados mediante el CISS. Todos los análisis se realizaron con el paquete estadístico SPSS 8.0.

\section{RESULTADOS}

Lo primero que pretendíamos fue determinar cuál era el patrón de personalidad 
de los pacientes de nuestra muestra mediante el MCMI-II. En cuanto a las escalas básicas de la personalidad obtuvimos puntuaciones elevadas en las escalas esquizoide $(X=76,96)$, dependiente $(X=70,07)$, fóbica $(X=73,50)$ y autodestructiva-masoquista $(X=63,96)$. Analizando las escalas patológicas de la personalidad obtuvimos puntuaciones por encima de la media en las escalas esquizotípicas $(X=70,39)$ y paranoide. $(X=60,11)$. En cuanto a los síndromes clínicos se obtuvieron valores significativos en el trastorno delirante $(X=65,00)$, psicótico $(X=61,04)$ y abuso de alcohol $(X=60,36)$. Estos datos coinciden con el perfil de paciente psicótico descrito en el manual del MCMI-II. (TEA, 1999).

Inicialmente, con el objetivo de realizar una selección, se calcularon las correlaciones simples entre las variables objeto de nuestro estudio. Los resultados obtenidos quedan reflejados en la Tabla 1.

Posteriormente, con el fin de averiguar si las variables de afrontamiento estudiadas tenían capacidad predictiva sobre las puntuaciones obtenidas en las escalas de personalidad y síndromes clínicos del MCMI-II, se realizaron análisis de regresión múltiple, considerando la puntuación obtenida en cada una de las escalas del MCMI-II como variable dependiente y las puntuaciones obtenidas en cada estrategia de afrontamiento como variables independientes. (Tabla 2).

Los resultados nos indican que el afrontamiento orientado a la tarea aparece como predictor significativo de Pensamiento psicótico y Depresión mayor. El afrontamiento orientado hacia la emoción aparece como predictor significativo de la escala Esquizoide. El afrontamiento orien-

Tabla 1. Correlaciones entre las estrategias de afrontamiento y las escalas del MCMI-II

\begin{tabular}{|c|c|c|c|c|}
\hline Escalas Clínicas & Tarea & Emoción & $\begin{array}{l}\text { Evitación- } \\
\text { Distracción }\end{array}$ & $\begin{array}{l}\text { Evitación- } \\
\text { Diversión }\end{array}$ \\
\hline Esquizoide &,$- 347^{\star}$ &, $348^{*}$ & &,$- 483^{* *}$ \\
\hline Fóbica & &, $442^{\star \star}$ &, $353^{*}$ & \\
\hline Histriónica & & & &, $391^{\star}$ \\
\hline Antisocial & & &, $341 *$ & \\
\hline Pasivo-Agresiva & & &, $336^{\star}$ & \\
\hline Autodestructiva-Masoquista & &, $385^{\star}$ & & \\
\hline Esquizotípica & &, $349^{*}$ &, $428 * \star$ & \\
\hline Límite & & &, $363^{*}$ & \\
\hline Paranoide & & &, $416^{*}$ & \\
\hline Ansiedad & & &, $589^{* *}$ & \\
\hline Histeriforme & & & $.550^{\star \star}$ & \\
\hline Hipomanía & & &, $425 * *$ & \\
\hline Distimia & &, $336^{*}$ &, $331^{*}$ & \\
\hline Abuso Alcohol & & &, $376^{\star}$ & \\
\hline Pensamiento Psicótico & & &, $548^{\star \star}$ & \\
\hline Depresión Mayor & &, $373^{*}$ &, $600 * *$ & \\
\hline Trastorno Delirante & & &, $530^{\star \star}$ & \\
\hline
\end{tabular}

(**) La correlación es significativa al nivel 0,01 (Unilateral)

(*) La correlación es significativa al nivel 0,05 (Unilateral) 
Tabla 2. Resultados obtenidos de los análisis de regresión múltiple (pasos sucesivos) realizados entre las Estrategias de Afrontamiento (V.I) y las Escalas Clínicas del MCMI-II (V.D)

\begin{tabular}{lcccc}
\hline Escalas clínicas & Estrategias afrontamiento & Beta & $\mathrm{R}^{2}$ & $\mathrm{p}$ \\
\hline Esquizoide & Evitación-Diversión & $-2,437$ &, 234 &, 007 \\
Fébición & Emoción & 0,667 &, 352 &, 003 \\
Histriónica & Evitación-Diversión & 1,280 &, 195 &, 015 \\
Autodestructiva-Masoquista & Evitación-Distracción & 1,734 &, 153 &, 033 \\
Esquizotípica & Evitación-Distracción & 1,881 &, 148 &, 036 \\
Límite & Evitación-Distracción & 1,689 &, 184 &, 018 \\
Paranoide & Evitación-Distracción & 1,395 &, 132 &, 049 \\
Ansiedad & Evitación-Distracción & 2,250 &, 173 &, 022 \\
Histeriforme & Evitación-Distracción & 1,651 &, 347 &, 001 \\
Hipomanía & Evitación-Distracción & 1,350 &, 181 &, 002 \\
Abuso de Alcohol & Evitación-Distracción & 1,330 &, 141 &, 041 \\
Pensamiento Psicótico & Evitación-Distracción & 2,458 &, 300 &, 002 \\
Trastorno Delirante & Tarea & $-0,839$ &, 422 &, 001 \\
Depresión Mayor & Evitación Distracción & 1,252 &, 281 &, 003 \\
\hline
\end{tabular}

tado hacia la evitación - diversión aparece como predictor significativo de las escalas Esquizoide e Histriónica. Por último, el afrontamiento orientado a la evitación distracción aparece como predictor de las escalas Paranoide, Autodestructiva-Masoquista, Esquizotípica, Límite, Ansiedad, Histeriforme, Hipomanía, Abuso de alcohol, Pensamiento psicótico, Trastorno delirante y Depresión mayor.

\section{DISCUSIÓN}

En el manual del MCMI-II (TEA, 1999), el perfil de paciente psicótico refleja puntuaciones elevadas en las escalas esquizoide, fóbica y dependiente de la personalidad, así como en el trastorno psicótico y paranoide. Como era esperable, dicho perfil coincide con el obtenido por nuestra muestra compuesta por esquizofrénicos crónicos.

Como objetivo principal de nuestro estudio, nos planteamos determinar la relación existente entre las distintas estrategias de afrontamiento y las escalas evaluadas mediante el MCMI-II. Al igual que otros estudios, nuestros resultados reflejan relaciones significativas entre las estrategias de afrontamiento y aquellos escalas de personalidad donde los sujetos obtienen las puntuaciones más elevadas. (Vollrath et al., 1994; Kruedelbach et al., 1993).

En mayar medida eran las estrategias de afrontamiento centradas en la evitación las que se relacionaban con puntuaciones elevadas en diversas escalas del MCMI-II. Los pacientes utilizaban estrategias de afrontamiento centradas en la evitación de situaciones problemáticas, principalmente realizando otras actividades (distracción) y en menor medida recurriendo a otras personas (diversión). Los datos también reflejan que dicha estrategia es muy poco utilizada por los sujetos que obtienen puntuaciones elevadas en la escala esquizoide de la personalidad. Dichos resultados eran espera- 
bles ya que estos pacientes se caracterizan por un patrón de retraimiento y aislamiento social. ( APA, 1994; OMS, 1992). Por el contrario, tales sujetos al igual que los que obtienen puntuaciones elevadas en la escala fóbica, utilizan estrategias de afrontamiento centradas en la emoción, es decir, tienden a afrontar las distintas situaciones estresantes autoculpabilizándose, autopreocupándose, manifestando intensas reacciones emocionales que predominan sobre un análisis lógico de la situación problemática.

Por último, en relación a los síndromes clínicos, los resultados muestran una correlación negativa entre aquellos participantes con puntuaciones elevadas en los síndromes clínicos graves ( pensamiento psicótico y depresión mayor) y la utilización de estrategias de afrontamiento centradas en la tarea. Estos resultados son coincidentes con los de otras investigaciones que establecen una relación negativa entre estrategias de afrontamiento positivas y diversas patologías.(Vollrath et al., 1998).

Los pacientes con menor grado de patología utilizan mas a menudo estrategias de afrontamiento centradas en la tarea y bajo afrontamiento emocional, mientras que los que informan más síntomas y deterioros muestran mayor tendencia al afrontamiento no centrado en el problema (evitación) con un bajo grado de esfuerzo cognitivo para controlar las reacciones emocionales que se pueden derivar de sus experiencias. (Wiedl y Schottner, 1991). Nuestros datos reflejan datos similares.

Como conclusión, las estrategias de afrontamiento es uno de los factores que contribuye al curso de los trastornos de la personalidad y otros síndromes clínicos. Por todo ello, el entrenamiento en estrategias de afrontamiento adecuadas debe ser una meta a conseguir en el tratamiento psicosocial de personas con patología psíquica.

\section{REFERENCIAS BIBLIOGRÁFICAS}

American Psychiatric Association (APA) (1994). Diagnostic and Statiscal Manual of Mental Disorder ( $4^{g}$ ed.) Washington: DC. (Edición española, Barcelona: Masson, 1995).

Beck, A.T., Freeman, A. et al. (1990). Cognitive therapy of personality disorders. New York: Guilford.

Bechdolf, A., Halve, S., Schultze-Lutter, F., y Klosterkotter, J. (1998). Self-experienced vulnerability, prodromic symptoms and coping strategies before schizophrenic and affective episodes. Fortschritteder Neurologieund Psychiatrie, 66, 378386.

Endler, N.S., y Parker, J.D.A. (1990). Multidimensional assessment of coping: A critical evaluation. Journal of Personality and Social Psychology, 58, 844-854.

Endler, N. S., y Parker, J.D.A. (1990). Coping Inventory for Stressful Situations. Versión española de Sánchez Elvira, A. 1996. No publicado.

Folkman, S. y Lazarus, R.S. (1980). An analysis of coping in a middle-aged community sample. Journal of Health and Social Bahavior, 21, 219-239.

Kruedelbach, N., McCormick, R.A., Schulz, S.C., y Grueneich, R. (1993). Impulsivity, coping styles and triggers for craving in substance abusers with borderline personality disorder. Journal of Personality Disorders, 7, 214-222.

Lazarus, R.S., y Folkman, S. (1984). Stress, appraisal, and coping. New York: Springer.

Middelboe, T., y Mortensen, E.L. (1997). Coping strategies among the long-term mentally ill: categorization and clinical determinants. Acta Psychiatrica Scandinavica, 96, 188-194.

Millon, T. (1981). Disorders of Personality. DSM-III: Axis II. New York: Wiley.

Millon, T. (1987). Millon Clinical Multiaxial Inventory-II. Versión española de Ávila Espada, A. y Jiménez, F. TEA Ediciones, Madrid 1998.

Millon, T. (1991). Manual del Inventario Clinico Multiaxial de Millon (MCMI-II). TEA Ediciones, Madrid 1999. 
Vollrath, M., Alnaes, R., y Torgersen, S. (1994). Coping and MCMI.II personality disorders. Journal of Personality Disorders, 8, 53-64.

Vollrath, M., Alnaes, R., y Torgersen, S. (1998). Coping styles predict change in personality disorders. Journal of Personality Disorders, 12, 198-209.

Van den Bosch, RJ., y Rombouts, RP. (1997).

Coping and cognition in schizophrenia and depression. Comprehensive Psychiatry, 38, 341-344.

Wield, R. y Schottner, B. (1991). Coping with symptoms related to schizophrenia. Schizophrenia Bulletin, 17, 525-538.

World Health Organization. (1992). The ICD10 Classification of Mental and Bahavioural Disorders. Diagnostic Criteria for Research. Geneva: WHO (Edición española, Madrid: Meditor, 1992). 